\title{
Scan, plan, print, practice, perform: Development and use of a patient-specific 3-dimensional printed model in adult cardiac surgery
}

\author{
Joshua L. Hermsen, MD, ${ }^{\mathrm{a}}$ Thomas M. Burke, PhD, ${ }^{\mathrm{b}}$ Stephen P. Seslar, MD, PhD, ${ }^{\mathrm{c}, \mathrm{d}}$ David S. Owens, MD, ${ }^{\mathrm{d}}$ \\ Beth A. Ripley, MD, PhD, ${ }^{\mathrm{e}}$ Nahush A. Mokadam, $\mathrm{MD},{ }^{\mathrm{a}}$ and Edward D. Verrier, $\mathrm{MD}^{\mathrm{a}}$
}

\begin{abstract}
Objective: Static 3-dimensional printing is used for operative planning in cases that involve difficult anatomy. An interactive 3D print allowing deliberate surgical practice would represent an advance.

Methods: Two patients with hypertrophic cardiomyopathy had 3-dimensional prints constructed preoperatively. Stereolithography files were generated by segmentation of chest computed tomographic scans. Prints were made with hydrogel material, yielding tissue-like models that can be surgically manipulated. Septal myectomy of the print was performed preoperatively in the simulation laboratory. Volumetric measures of print and patient resected specimens were compared. An assessment tool was developed and used to rate the utility of this process. Clinical and echocardiographic data were reviewed.
\end{abstract}

Results: There was congruence between volumes of print and patient resection specimens (patient $1,3.5 \mathrm{~cm}^{3}$ and $3.0 \mathrm{~cm}^{3}$, respectively; patient $2,4.0 \mathrm{~cm}^{3}$ and $4.0 \mathrm{~cm}^{3}$, respectively). The prints were rated useful (3.5 and 3.6 on a 5-point Likert scale) for preoperative visualization, planning, and practice. Intraoperative echocardiographic assessment showed adequate relief of left ventricular outflow tract obstruction (patient 1,80 $\mathrm{mm} \mathrm{Hg}$ to $18 \mathrm{~mm} \mathrm{Hg}$; patient 2, $96 \mathrm{~mm} \mathrm{Hg}$ to $9 \mathrm{~mm}$ $\mathrm{Hg}$ ). Both patients reported symptomatic improvement (New York Heart Association functional class III to class I).

Conclusions: Three-dimensional printing of interactive hypertrophic cardiomyopathy heart models allows for patient-specific preoperative simulation. Resection volume relationships were congruous on both specimens and suggest evidence of construct validity. This model also holds educational promise for simulation of a low-volume, high-risk operation that is traditionally difficult to teach. (J Thorac Cardiovasc Surg 2017;153:132-40)

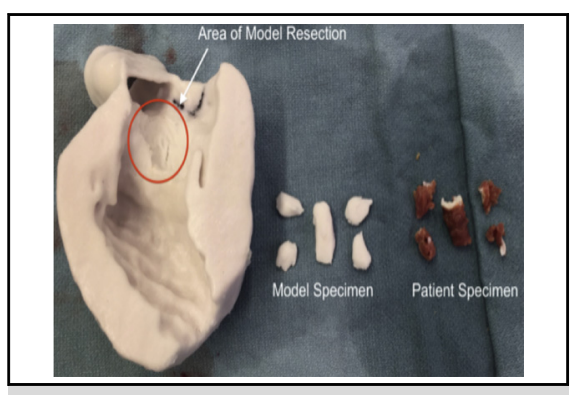

Three-dimensional print (after myectomy in sim lab) next to operative myectomy specimen.

Central Message
Interactive 3-dimensional prints allowing
patient-specific operative rehearsal of septal
myectomy represent a novel medical 3-
dimensional printing application.

Perspective

Medical 3-dimensional (3D) printing represents a potentially disruptive technology with myriad applications. To date, 3D printing applications in cardiothoracic surgery have served almost exclusively as adjuncts to conventional imaging. The development of an interactive 3D print allowing surgeons to practice patient-specific operations represents a novel medical 3D printing application.

See Editorial Commentary page 141.
Three-dimensional (3D) printing is an emerging technology in medicine with a yet-to-be-defined role. To date, 3D

\footnotetext{
From the Divisions of ${ }^{\mathrm{a}}$ Cardiothoracic Surgery and ${ }^{\mathrm{d}}$ Cardiology, and ${ }^{\mathrm{e}}$ Department of Radiology, University of Washington; ${ }^{\mathrm{b}}$ Thomas Burke PhD Consultant, LLC, Bothell; and ${ }^{\mathrm{c}}$ Pediatric Heart Center, Seattle Children's Hospital, Seattle, Wash.

Read at the 42nd Annual Meeting of The Western Thoracic Surgical Association, Waikoloa, Hawaii, June 22-25, 2016.

Received for publication June 22, 2016; revisions received Aug 2, 2016; accepted for publication Aug 11, 2016; available ahead of print Sept 17, 2016.

Address for reprints: Joshua L. Hermsen, MD, Division of Cardiothoracic Surgery, University of Washington, Box 356310, Suite AA115, 1959 NE Pacific St, Seattle, WA 98195 (E-mail: hermsej1@uw.edu).

$0022-5223 / \$ 36.00$

Copyright (c) 2016 by The American Association for Thoracic Surgery

http://dx.doi.org/10.1016/j.jtcvs.2016.08.007
}

printing in cardiac surgery has been most often used by congenital heart surgeons in an attempt better to understand complex anatomy, determine feasibility of corrective repair, and develop a priori operative strategies. ${ }^{1,2}$ Although the advantages afforded by static 3D-printed models are hard to quantify objectively, surgeons report subjective benefit,

Scanning this QR code will take you to a video for the article. 


\section{Abbreviations and Acronyms \\ 3D $=3$-dimensional \\ LVOTO = left ventricular outflow tract obstruction \\ HCM = hypertrophic cardiomyopathy \\ TEE = transesophageal echocardiography \\ NYHA $=$ New York Heart Association \\ $\mathrm{SAM}=$ systolic anterior motion}

and in some cases a 3D print may change the operative approach.

Three-dimensional printing would seem to hold promise for the surgeon to create patient-specific anatomy on which to practice a planned operation, yet there are only 2 case reports of this in the medical literature. ${ }^{3,4}$ This lack is despite a growing body of simulation research demonstrating the benefits of such deliberate practice. ${ }^{5}$ This is likely driven in part by current limitations in printing to scale with materials that can be manipulated (cut, dissected, retracted, sutured) in ways meaningful to a surgeon. Although 3D prints are physical models, they have therefore largely served only as adjuncts to the standard 2-dimensional imaging modalities from which they are derived.

Surgical myectomy for treatment of left ventricular outflow tract obstruction (LVOTO) in patients with hypertrophic cardiomyopathy (HCM) is a conceptually simple yet challenging $3 \mathrm{D}$ operation performed predominately by adult cardiac surgeons. Because of the exclusively resectional, as opposed to reconstructive, nature of septal myectomy, it lends itself to operative simulation with a current 3D printing technology.

The process described in this study was developed to allow patient specific planning and quantitative operative rehearsal for patients undergoing myectomy for treatment of HCM with LVOTO. Institutional review board waiver was granted in accordance with established university guidelines.

\section{MATERIALS AND METHODS \\ Computed Tomographic Scanning}

Cardiac computed tomographic scans were obtained on a Revolution scanner (GE Medical Systems, Chicago, Ill) with 160-mm detector coverage in axial mode with standard low-dose techniques and a noise index of 30. Electrocardiographic gating was used, and images were prospectively acquired at $60 \%$ to $80 \%$ of the RR interval (during diastole) during a single cardiac cycle. Images were then reconstructed at $0.625-\mathrm{mm}$ slice thickness with $70 \%$ adaptive statistical iterative reconstruction $\mathrm{V}$ at the best time point in the RR interval (that with the least cardiac motion). A 3-phase contrast bolus was used as follows: 100\% iohexol (Omnipaque 350 ) at $5 \mathrm{~mL} / \mathrm{s}$ for $50 \mathrm{~mL} ; 70 \%$ Omnipaque 350 and $30 \%$ normal saline solution at $5 \mathrm{~mL} / \mathrm{s}$ for $50 \mathrm{~mL}$; and $100 \%$ normal saline solution at $5 \mathrm{~mL} /$ $\mathrm{s}$ for $50 \mathrm{~mL}$. This 3-phase bolus administration ensured opacification of both the right and left sides of the heart at the time of scanning, which was necessary for segmentation of the intraventricular septum. In addition, the saline solution chaser ensured that streak artifact from contrast entering the right side of the heart was minimized.

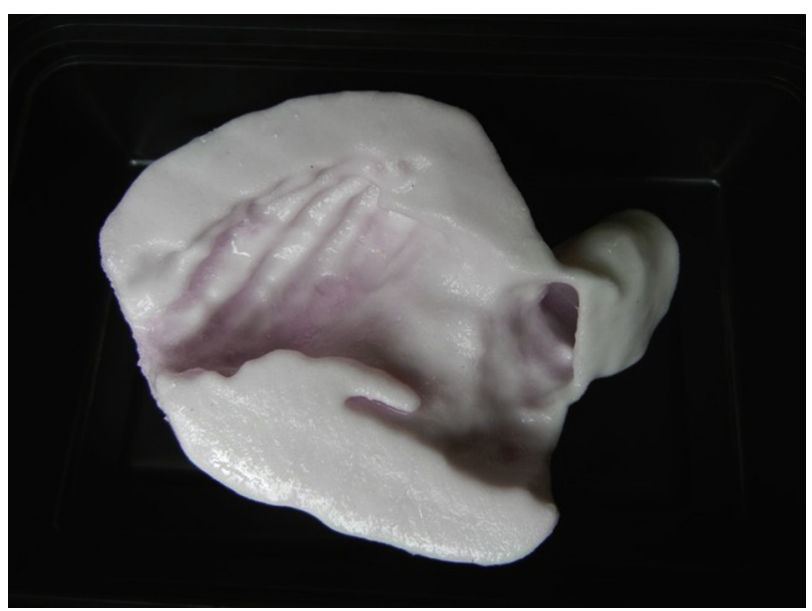

FIGURE 1. Photograph of a 3-dimensional printed model of heart with hypertrophic cardiomyopathy used in this study before any manipulation. The same print is shown in Figure 4 after manipulation.

\section{Segmentation and Digital Modeling}

Segmentation was performed by an engineer (T.M.B.) with cardiac 3D printing experience. DICOM formatted raw image data was reviewed with a surgeon (J.L.H.) to identify key anatomic features and regions of interest. Multiplanar image reconstruction was performed using the OsiriX opensource DICOM viewer.

Segmentation to yield a stereolithography (.stl) file, was performed with open-source code ITK-Snap as a parent program, augmented with useradded software routines from open-source code ITK and VTK libraries. ${ }^{7-9}$ When the ratio of signal to noise between tissue and the contrast pool was high, intensity-based region competition segmentation was performed to isolate cardiac anatomy. When computed tomographic speckle noise was high, gradient- or edge-based algorithms were used with manual segmentation. Edge-based algorithms were used to segment key features, including the aorta, anterior mitral valve leaflet, ventricular septum, papillary muscles, and chordae tendineae.

Digital mesh construction and optimization was performed in ITK-Snap to create an initial digital mesh. Cut-plane processing was used to eliminate unnecessary anatomy and provide a clear view of the ventricular septum. Final model generation, surface conditioning, and triangle reduction were accomplished with a custom software program that was based on P3D code libraries. $^{10}$

\section{D Printing}

The optimized digital mesh in the .stl file format was exported to a $\mathrm{Z}$ Corporation model 310 3D printer (3D Systems, Inc, Rock Hill, SC) for manufacture. Proprietary materials were used to construct a $3 \mathrm{D}$ porous scaffold that matched the digital mesh to $\pm 1 \mathrm{~mm}$ globally. The porous scaffold was then infused with silicone and a blend of 2 hydrogels. Silicone provided a flexible protective layer intended to extend the life of the model and facilitate handling and manipulation. A proprietary blend of 2 hydrogels was infused into the scaffold to mimic the feel of myocardial tissue. The completed models (Figure 1) were stored in a food-grade solution of propylene glycol and deionized water before and after use.

\section{Operative Rehearsal in Simulation Laboratory}

The day before the scheduled operation, the operating surgeon (J.L.H.) performed a myectomy on the 3D print. This was done in a simulation laboratory operating room furnished with an operating table and surgical lighting. The print was positioned in an anatomic position (accomplished by propping the print on rolled towels) within the cavity of a chest mannequin (Figure 2). Surgical drapes and a chest retractor were used to recreate the 


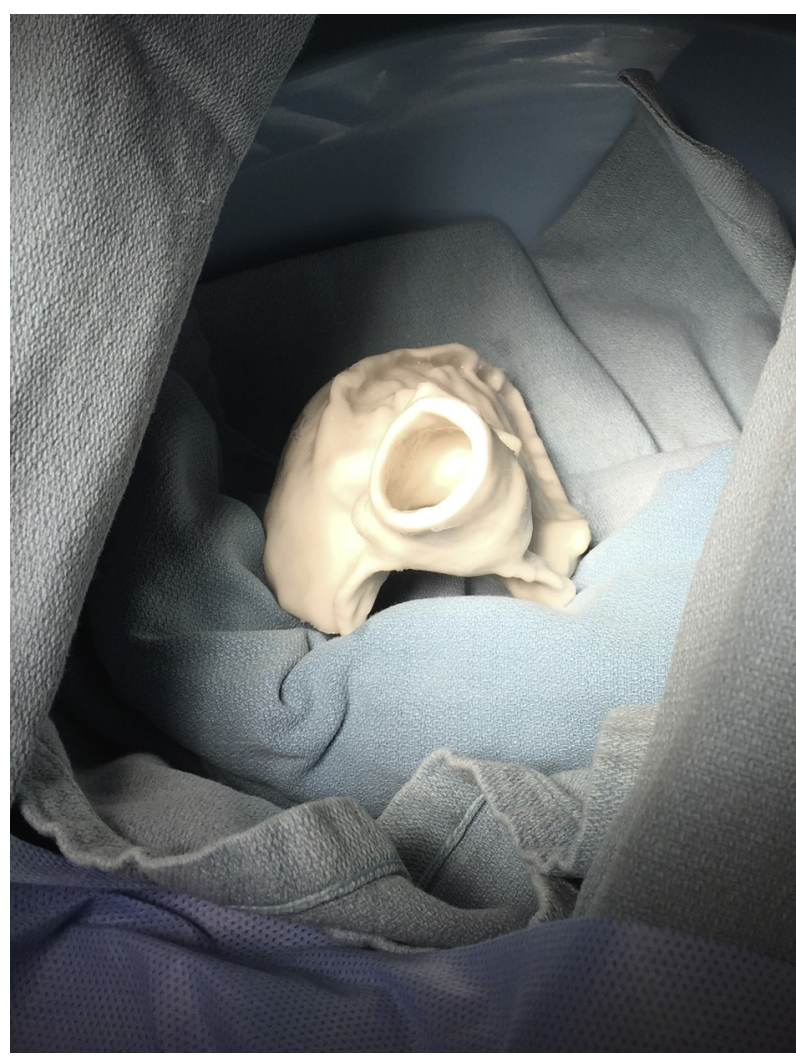

FIGURE 2. Photograph of a 3-dimensional print positioned for operative rehearsal in the well of a chest mannequin.

surgical field as much as possible. The operating surgeon wore an operating gown, mask, gloves, extended-field $3.5 \times$ magnification loupes (Designs for Vision, Ronkonkoma, NY), and a surgical headlight with highdefinition video camera (Sunoptic Technologies, Jacksonville, Fla). The myectomy of the 3D print was performed through the aorta with a No. 11 blade (Becton-Dickinson, Franklin Lakes, NJ) mounted on a long knife handle (Composite Figure 3). After the initial resection of several pieces, the print was removed from the mannequin and examined from all angles. Additional resection was done after this assessment in an attempt to perform the "optimal" myectomy, designed in part on the basis of the nonanatomic viewpoints afforded by the $3 \mathrm{D}$ print.

\section{Resection Volume Measurement}

A $50-\mathrm{mL}$ glass graduated cylinder was filled with $20 \mathrm{~mL}$ of water and placed on a level surface. All pieces resected from the 3D print (initial and subsequent) were dropped into the cylinder, and the change in level measured at the meniscus was recorded. The same graduated cylinder was steam sterilized and used similarly to measure the volume of septal muscle resected from the patient the next day. The surgeon was blind to the patient resection specimen volume until the operation had been completed (ie, the patient's resection was not guided by sequential intraoperative volume measurement to reach the $3 \mathrm{D}$ print resection volume). The resected 3D print was present in the operating room on a nonsterile Mayo stand for visual reference during the patient resection. After the operation, the model and patient specimens were placed next to each other and photographed for visual comparison (Figure 4).

Because the resected 3D print and muscle specimens both sank to the bottom of the graduated cylinder, the rise in milliliters of the liquid level was necessarily equal to the volume of the specimens in cubic centimeters, regardless of any differences in density.

\section{Assessment Tool}

An assessment tool was designed to evaluate the performance of the digital reconstruction and 3D prints (Figures 5 and 6 ).

\section{Transesophageal Echocardiography LVOT Gradient Measurement and Visualization}

Intraoperative transesophageal echocardiography (TEE) was standard. Transgastric views were used to measure LVOT velocity by continuouswave Doppler before and after myectomy. Peak gradients were calculated from optimal spectral Doppler envelopes and velocities calculated according to the formula $4 \mathrm{~V}^{2}$.

\section{RESULTS \\ Patient 1}

Patient 1 was a 52-year-old obese (body mass index, $47 \mathrm{~kg} / \mathrm{m}^{2}$ ) woman with HCM diagnosed at the time of symptom onset 19 years before the operation. She reported New York Heart Association (NYHA) class IIIb heart failure symptoms without angina. Transthoracic echocardiographically derived resting LVOT gradient measured $93 \mathrm{~mm} \mathrm{Hg}$, and the same measurement by TEE in the operating rom was $80 \mathrm{~mm} \mathrm{Hg}$. Systolic anterior motion (SAM) of the mitral valve was present resulting in moderate mitral regurgitation. Myectomy of the 3D print, performed in the simulation laboratory operating room the day before the patient's scheduled operation, yielded $3.5 \mathrm{~cm}^{3}$ of resected specimen.

Septal myectomy was performed through a transverse aortotomy extended into the noncoronary sinus. A total specimen of $3.0 \mathrm{~cm}^{3}$ of muscle was resected. Accessory chordae from the anterior mitral valve leaflet to the ventricular septum were also excised. Cardiopulmonary bypass time was 61 minutes, and cardiac ischemic time was 34 minutes.

Postoperatively, the TEE-derived peak and mean gradients were $18 \mathrm{~mm} \mathrm{Hg}$ and $8 \mathrm{~mm} \mathrm{Hg}$, respectively. SAM was absent, and mitral regurgitation was trace to mild. The patient's recovery was uneventful except for paroxysmal atrial fibrillation. The patient has reported improvement in symptoms to NYHA functional class 1 .

The myectomy for HCM 3D printing assessment tool, completed after the patient operation, yielded an averaged rating of 3.5 on a Likert scale from 1 to 5 (Figure 5).

\section{Patient 2}

Patient 2 was a 35 -year-old man with cerebrotendinous xanthomatosis and HCM diagnosed 6 years before the operation. He reported NYHA class III heart failure symptoms and frequent near syncope. Resting LVOT gradient measured in the catheterization laboratory was $4 \mathrm{~mm} \mathrm{Hg}$, which increased to $144 \mathrm{~mm} \mathrm{Hg}$ with physiologic provocation. SAM of the mitral valve was present, with provocation resulting in moderate mitral regurgitation. Measurement of the LVOT gradient by TEE in the operating room was $93 \mathrm{~mm} \mathrm{Hg}$. Myectomy of the 3D print performed in the 


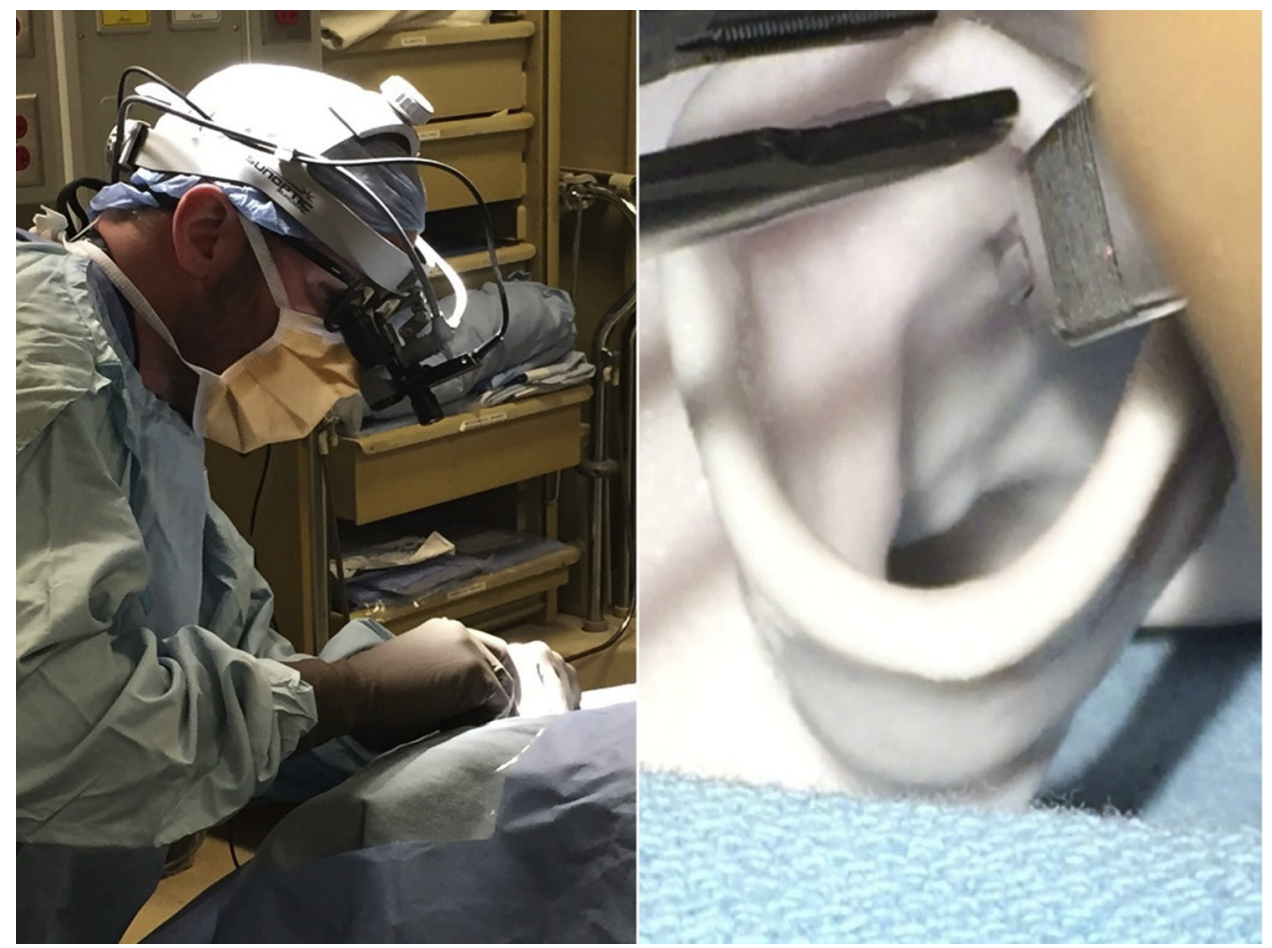

FIGURE 3. Composite photograph showing the operating surgeon during rehearsal in the simulation laboratory (left) and the operating surgeon's view of myectomy on a 3-dimensional print (right).

simulation laboratory operating room the day before the patient's scheduled operation yielded $4.0 \mathrm{~cm}^{3}$ of resected specimen (Video 1).

Septal myectomy was performed through a transverse aortotomy extended into the noncoronary sinus. A $4.0 \mathrm{~cm}^{3}$ specimen of muscle was resected. An iatrogenic ventricular septal defect was repaired through the right atrium, and an epicardial permanent pacemaker was placed for complete heart block. Cardiopulmonary bypass time was 226 minutes, and cardiac ischemic time was 152 minutes.

Postoperatively, the TEE-derived peak gradient was $9 \mathrm{~mm} \mathrm{Hg}$. SAM was absent, and mitral regurgitation was

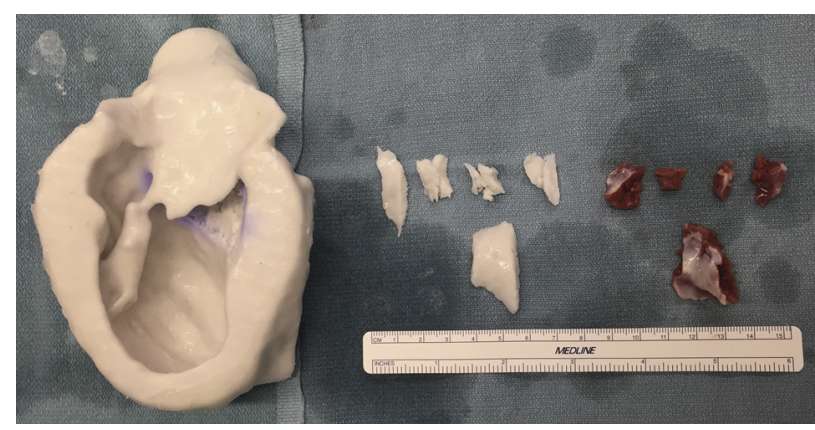

FIGURE 4. Photograph in the operating room of the 3-dimensional print and its myectomy specimens next to the actual myectomy specimen from same patient for comparison. mild. The patient's recovery was uneventful. He has reported improvement in symptoms to NYHA functional class 1 without near syncope.

The myectomy for HCM 3D printing assessment tool, completed after the patient operation, yielded an overall rating of 3.6 on a Likert scale from 1 to 5 (Figure 6).

\section{DISCUSSION}

Medical 3D printing is, in many ways, a solution in search of problems. The potential applications, in surgical specialties alone, are innumerable. To date, however, 3D prints have been most commonly used as tangible adjuncts to the imaging modalities from which they are derived. ${ }^{11,12}$ Although the potential benefits to surgeons of a tangible, physical model are intuitively clear, such as visualization of complex anatomy, most such models do not allow surgeons actually to practice the planned operation. This experience sought to take the use of $3 \mathrm{D}$ prints in cardiothoracic surgery a step further, providing the surgeon an opportunity for patient-specific deliberate practice of a planned operation. Operative rehearsals with patient-specific 3D prints may reveal to a surgeon potential pitfalls, a preferred approach, or optimal instrumentation, at the same time providing a preview of anatomic nuance and potentially forming muscle memory.

This study describes a process used for 2 patients undergoing septal myectomy for HCM. In this scheme, the 


\section{D Print - HCM/Myectomy 3D Printing Assessment Tool}

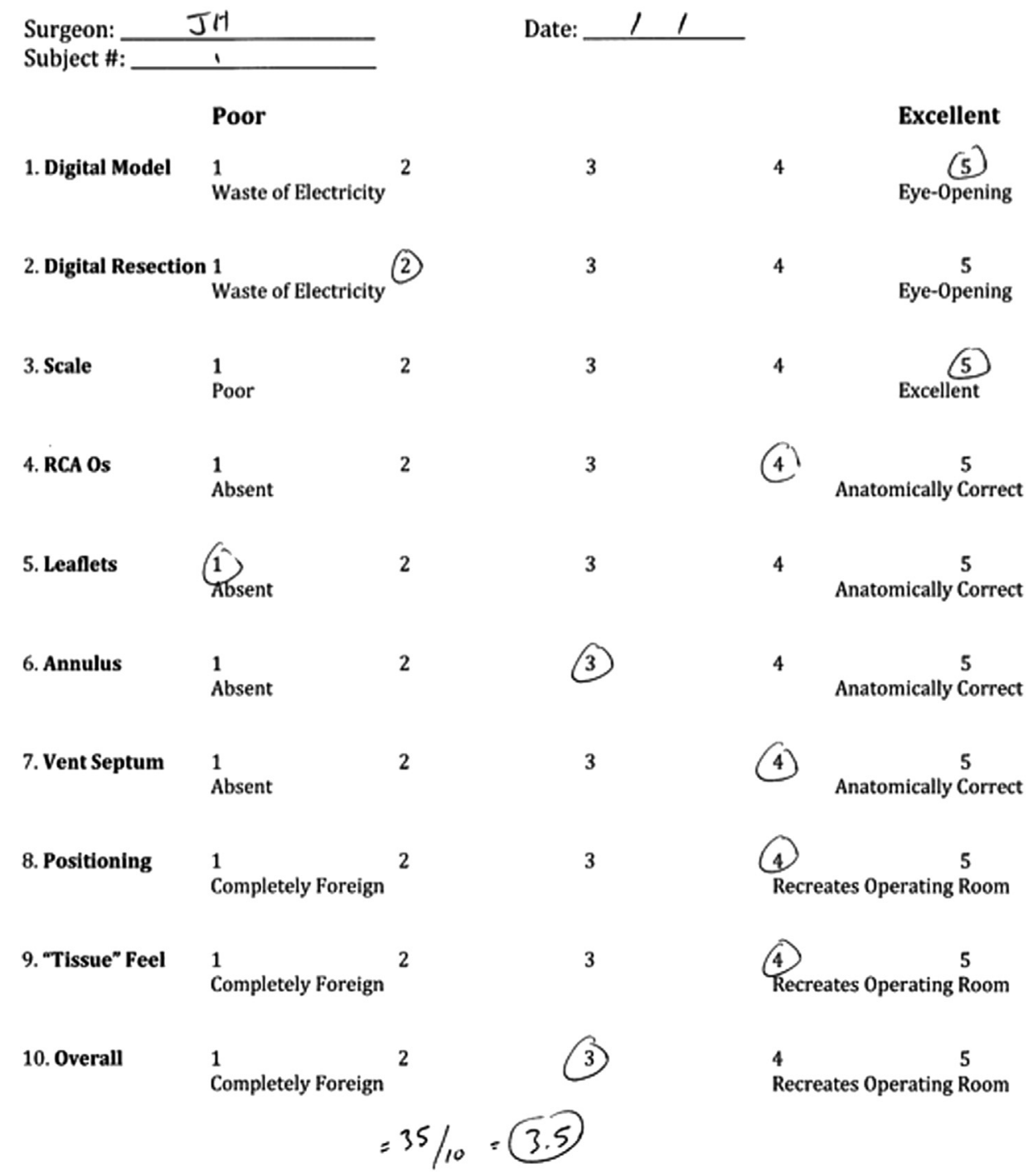

FIGURE 5. Assessment tool used to rate the usefulness of information and experience gained from the 3-dimensional (3D) printing process, including operative rehearsal on the 3-dimensional print. Simulator assessment tool for patient 1 is shown. $H C M$, Hypertrophic cardiomyopathy; $R C A$, right coronary artery.

surgeon was provided 2 tools not previously available: an interactive digital model (Figure 7) and a physical 3D print (Figure 1). The digital model is a byproduct of the segmentation process necessary to create a $3 \mathrm{D}$ print from computed tomographic scan data and is infinitely manipulable. The heart can be sectioned in any plane, and the image can be rotated on the screen in all axes. The ability to section, rotate, and view a 3-dimensional representation of the heart and ventricular septum in nontraditional planes as well as in sagittal, coronal, and axial planes was subjectively useful according to the assessment tool. The surgeon was also able to perform a virtual myectomy with the segmentation software. This was actually less useful than anticipated, however, because the graphical user interface for this function was not surgically intuitive. In addition, the volume of the digital resection was not calculable, so a comparison could not be made with the resection volumes from the 3D print and the patient.

The 3D-printed patient-specific model enabled deliberate practice of the planned operation. The technical simplicity of myectomy, the nondelicate nature of septal muscle, and the lack of tissue-plane dissection necessary to expose the operative target all favor the use of current technology 3D prints for this purpose. The model was sufficiently realistic in terms of operative exposure and anatomy. The overall assessment tool scores were modest, but they were 


\section{D Print - HCM/Myectomy 3D Printing Assessment Tool}

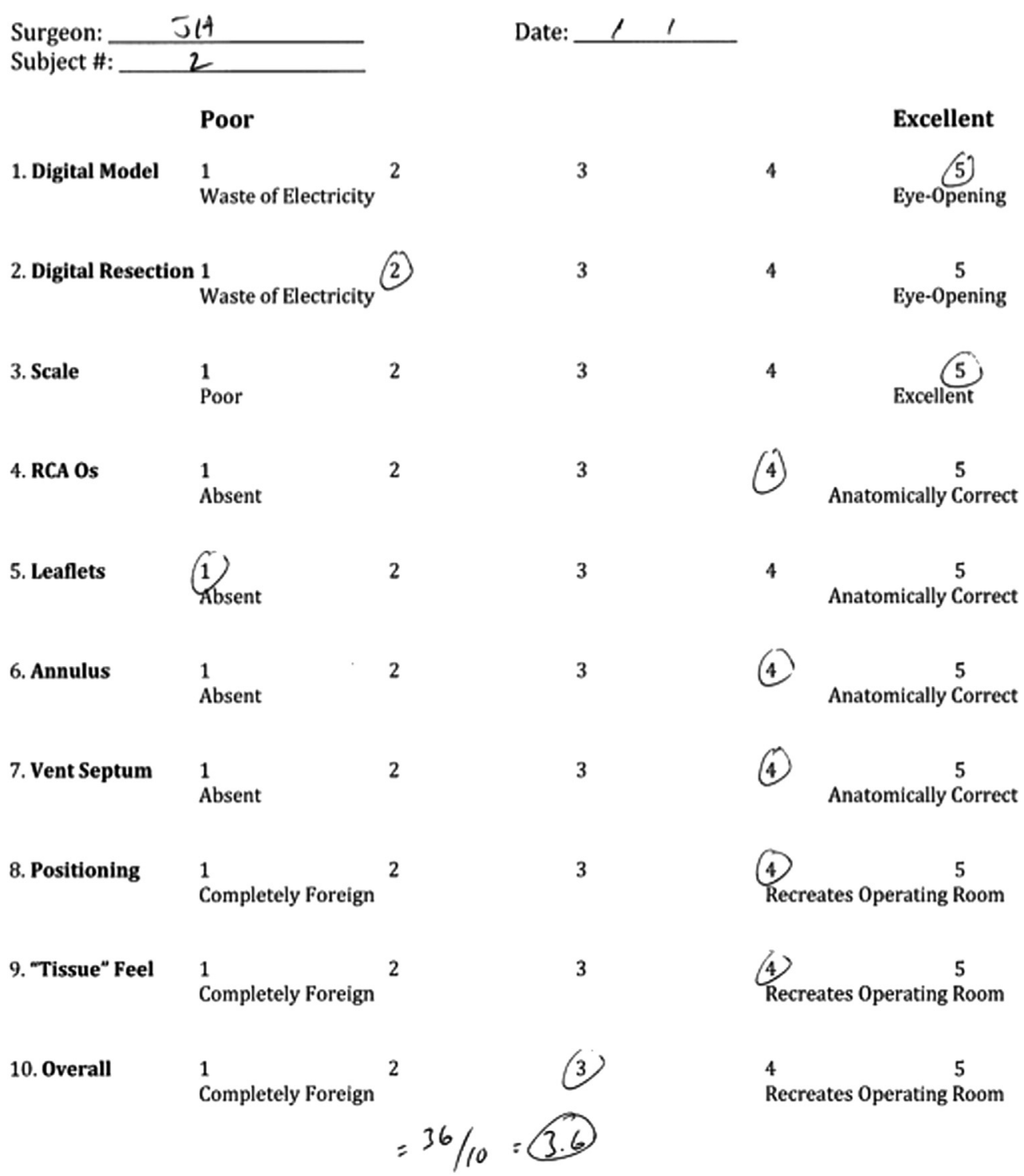

FIGURE 6. Assessment tool used to rate the usefulness of information and experience gained from the 3-dimensional (3D) printing process, including operative rehearsal on 3-dimensional print. Simulator assessment tool for patient 2 is shown. HCM, Hypertrophic cardiomyopathy; RCA, right coronary artery.

reasonable for the first iteration of a completely new simulation platform. The hydrogel print medium used does a reasonable job approximating the consistency of cardiac muscle. It is overall firmer and less deformable than myocardium but is nicely homogenous and possesses a consistency that provides a "connective tissue" feel. The model allowed realistic creation of the operative resection experience. In both cases, a dominant specimen was resected first and multiple smaller pieces were resected subsequently, just as is usual practice in the operating room. Importantly, the 3D print allowed the performance of a more nearly ideal resection, because the print could be picked up, turned over, viewed and palpated from all angles, and resected further until the resultant septal anatomy appeared completely optimal.

The 3D prints were constructed on a 1:1 scale, which allowed the comparison of print and patient resection specimen volumes. The 1:1 scale also facilitated and reinforced any muscle memory from the resection performed on the print. The use of liquid displacement allowed the accurate measurement of materials with different densities because both were nonbuoyant (sank to the bottom of the liquid column and thus were completely submerged). The measured resection volume of the $3 \mathrm{D}$ print specimen was not used to guide patient resection; however, the general agreement of the volumes in both cases provides some signal that the 


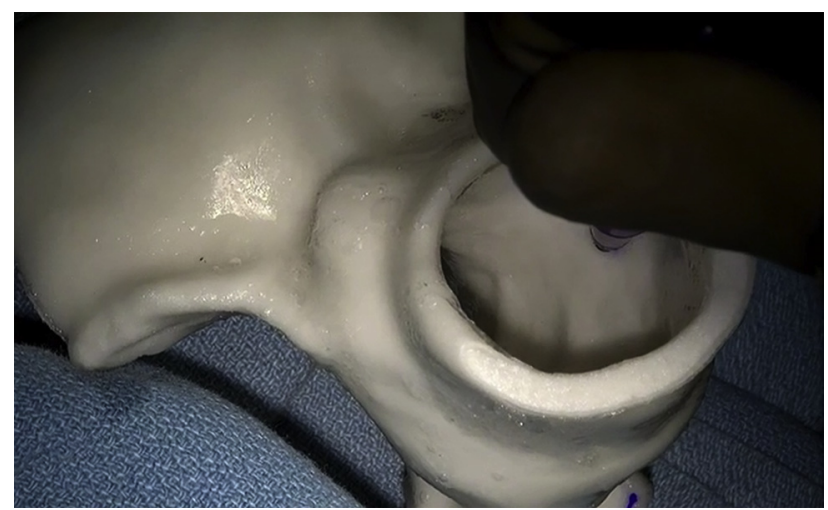

VIDEO 1. Video clip shows the surgeon's view during the myectomy performed on the 3-dimensional model for patient 2. Video available at: http:// www.jtcvsonline.org/article/S0022-5223(16)30937-0/addons.

print resection volume may be useful as a "goal" number to drive resection in the operating room. If the volume measured from an "optimal" resection done on the 3D print can inform a more complete patient myectomy, this 3D printing schema could help to foster improved surgical outcomes.

Mitral valvular and subvalvular abnormalities are common in this patient population and often contribute to LVOT obstruction, and 3D printing may be able to help define and understand them better preoperatively. ${ }^{13}$ The $3 \mathrm{D}$ print in the case of patient 2 revealed an abnormality involving the subvalvular mitral apparatus in which a papillary muscle inserted directly onto the leaflet (Figures 8 and

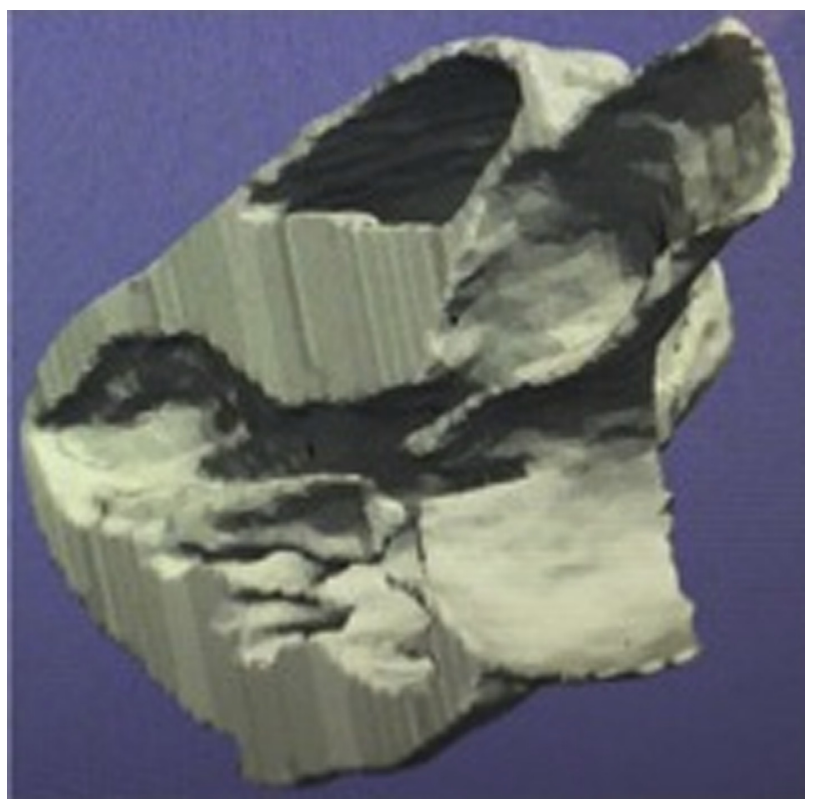

FIGURE 7. Screen shot of virtual 3-dimensional model produced during segmentation process.

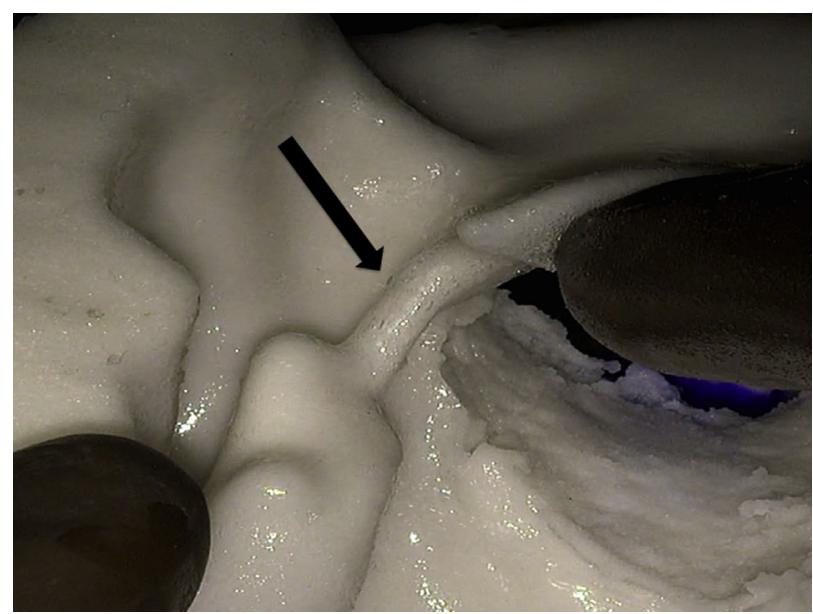

FIGURE 8. Photograph of a 3-dimensional print showing papillary muscle insertion directly onto the anterior mitral valve leaflet (arrow). The view is from the ventricular side looking out the left ventricular outflow tract.

9). Both patients, however, also had small, short accessory chordae with direct attachments to the ventricular septum that were not shown by the 3D print because of their very small size.

Demonstration of an objective or quantitative benefit of 3D prints used for patient-specific operative rehearsal of myectomy (or any other operation) may prove difficult. Contemporary surgical and patient outcomes with septal myectomy for the treatment of LVOTO in HCM are already excellent, with dramatic improvement or even total alleviation of symptoms expected. ${ }^{14}$ This small experience demonstrates that $3 \mathrm{D}$ printing is unlikely to render surgical complications a thing of the past. In fact, some metrics of quality or improvement may correlate inversely when a $3 \mathrm{D}$ print is used for preoperative preparation. If deliberate practice on a print enhances anatomic understanding and

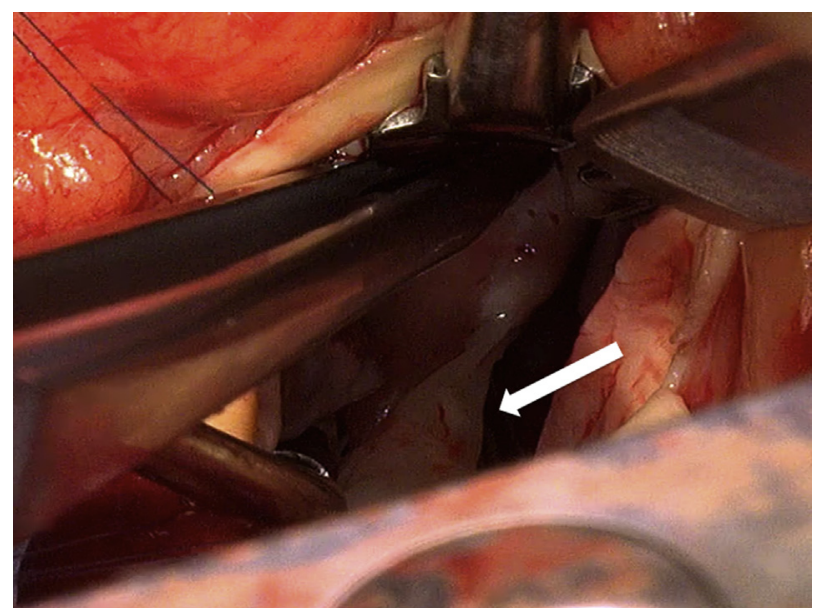

FIGURE 9. Intraoperative photograph showing papillary muscle insertion directly onto anterior mitral valve leaflet (arrow). The view is from the aortic side looking into the left ventricle. 
drives a more extensive operation, the operating room, bypass, and cardiac ischemic times could actually increase! This experience provides some proof of concept for patientspecific 3D printing and operative rehearsal, but more investigation is needed. For this specific application, short-term results such as echocardiographically derived LVOT area and longer-term results related to durability of symptom improvement and LVOTO recurrence rates would likely be needed to show the benefit of an incrementally more complete myectomy. Such a comparison would need a large number of subjects and multiple surgeons to be powered adequately.

The use of 3D prints for operative rehearsal of septal myectomy may have a higher yield in extreme or unusual cases in which the surgeon confronts extremely abnormal anatomy. Deliberate practice using 1 or more 3D prints in the simulation laboratory in this instance would likely be rated as quite high yield. For young myectomy surgeons with little experience, operative simulation with patient-specific 3D prints could flatten the learning curve. Similarly, even for experienced surgeons who rarely perform myectomy through apical or transmitral approaches, the chance to practice the operation from an unfamiliar vantage point with a 3D print could have important utility.

Myectomy is a difficult operation for most surgeons to teach. In addition, in most academic institutions, myectomy is not a high-volume procedure. The visibility is poor for anyone but the operating surgeon, and the anatomy is difficult because it is not familiar to trainees from their experience with other operations. Added to these hurdles are the impactful specific complications of myectomy including heart block, ventricular septal defect, and injury to aortic and mitral valves. The ability for residents to learn and practice on 3D prints representing "real anatomy" and to compare resections done by attending and resident surgeons on identical 3D prints may enable a resident to demonstrate sufficient knowledge, skill, and confidence to learn and perform these rewarding cases while in training. This could be facilitated by the incorporation of a myectomy for HCM module into the Cardiac Surgery Simulation Curriculum. ${ }^{15}$ The use of a porcine heart, as is standardly used in the Cardiac Surgery Simulation Curriculum, is certainly a costattractive alternative. In fact, most pigs have fairly striking asymmetric basilar septal hypertrophy that would allow the performance of meaningful myectomy. For initial instruction with early-stage learners, the porcine heart may be a reasonable alternative; however, the patient-specific nature of the 3D print, especially when used in close temporal proximity to operation, is certainly superior for specific preparation. The potential ability to quantitate and compare specimens volumetrically is also a distinct advantage of a patient-based model versus animal model. Concomitant work is ongoing to ensure that the segmentation and printing process yields an accurate model that can be used quantitatively.

\section{Limitations}

This is a small, single-institution pilot experience in using patient specific 3D prints for deliberate practice and operative rehearsal. Although this schema provides proof of concept for the use of 3D prints to rehearse operations, limited conclusions or inferences can be made. In addition, although the combination of current technology and technical details of septal myectomy align well for this particular purpose, similar prints would not be particularly useful in simulating valve, coronary, or aortic surgery. Particularly thin structures (leaflets) or small structures (coronary arteries) are not yet printable in a way that is useful for surgeons to practice these operations. The simulator assessment scores indicate that this concept has the promise to be useful but needs improvement, which is ongoing. ${ }^{16}$

This technology involves time and monetary costs in addition to the standard care of patients undergoing myectomy. The additional clinician time to set up for, perform, and analyze the rehearsal myectomy was approximately 90 minutes. This time will likely decrease to 60 minutes or less as experience is gained and the process is streamlined. The monetary cost currently is US $\$ 400$ per print.

\section{CONCLUSIONS}

The model described in this article is unique in its primary purpose of allowing a surgeon to visualize and practice an operation preoperatively on a patient-specific 3D print. Combined with the growing experience and data regarding surgical simulation, 3D printing is poised to enable widespread patient-specific deliberate practice, akin to flight simulation in the aviation industry. Operative rehearsal with 3D prints may be meaningful to surgeons at all levels of skill and experience, depending on the specific circumstance. Three-dimensional printing technology may provide enhanced educational opportunities for trainees, especially for low-volume, low-visibility, high-risk operations such as septal myectomy.

\section{Conflict of Interest Statement}

T.M.B. is owner of a for-profit corporation that manufactures patient-specific heart models and received reimbursement for materials used in producing heart models for this study. All other authors have nothing to disclose with regard to commercial support.

\section{References}

1. Noecker AM, Chen JF, Zhou Q, White RD, Kopcak MW, Arruda MJ, et al.
Development of patient-specific three-dimensional pediatric cardiac models.
ASAIO J. 2006;52:349-53. 
2. Sodian R, Weber S, Markert M, Loeff M, Lueth T, Weis FC, et al. Pediatric cardiac transplantation: three-dimensional printing of anatomic models for surgical planning of heart transplantation in patients with univentricular heart. $J$ Thorac Cardiovasc Surg. 2008;136:1098-9.

3. Kiraly L, Tofeig M, Jha NK, Talo H. Three-dimensional printed prototypes refine the anatomy of post-modified Norwood-1 complex aortic arch obstruction and allow presurgical simulation of the repair. Interact Cardiovasc Thorac Surg. 2016;22:238-40.

4. Shiraishi I, Yamagishi M, Hamaoka K, Fukuzawa M, Yagihara T. Simulative operation on congenital heart disease using rubber-like urethane stereolithographic biomodels based on 3D datasets of multislice computed tomography. Eur J Cardiothorac Surg. 2010;37:302-6.

5. Hashimoto DA, Sirimanna P, Gomez ED, Beyer-Berjot L, Ericsson KA, Williams NN, et al. Deliberate practice enhances quality of laparoscopic surgical performance in a randomized controlled trial: from arrested development to expert performance. Surg Endosc. 2015;29:3154-62.

6. Rosset A, Spadola L, Ratib O. OsiriX: an open-source software for navigating in multidimensional DICOM images. J Digit Imaging. 2004;17:205-16.

7. Yushkevich PA, Piven J, Hazlett HC, Smith RG, Ho S, Gee JC, et al. User-guided $3 \mathrm{D}$ active contour segmentation of anatomical structures: significantly improved efficiency and reliability. Neuroimage. 2006;31:1116-28.

8. National Library of Medicine. Insight segmentation and registration toolkit. Available at: http://www.itk.org. Accessed April 21, 2015.

9. Martin K, Schroeder W, Lorensen B. Visualize your data with VTK. Available at: http://www.vtk.org. Accessed April 21, 2015.
10. Pacific Northwest National Laboratory. P3D. Available at: http://www.emsl.pnl. gov/nwgrid/. Accessed April 21, 2015.

11. Schmauss D, Juchem G, Weber S, Gerber N, Hagl C, Sodian R. Three-dimensional printing for perioperative planning of complex aortic arch surgery. Ann Thorac Surg. 2014;97:2160-3.

12. Dickinson KJ, Matsumoto J, Cassivi SD, Reinersman JM, Fletcher JG, Morris J, et al. Individualizing management of complex esophageal pathology using threedimensional printed models. Ann Thorac Surg. 2015;100:692-7.

13. Sherrid MV, Balaram S, Kim B, Axel L, Swistel DG. The mitral valve in obstructive hypertrophic cardiomyopathy: a test in context. J Am Coll Cardiol. 2016;67: 1846-58.

14. Sedehi D, Finocchiaro G, Tibayan Y, Chi J, Pavlovic A, Kim YM, et al. Longterm outcomes of septal reduction for obstructive hypertrophic cardiomyopathy. J Cardiol. 2015;66:57-62.

15. Feins RH, Burkhart HM, Coore DN, Conte JV, Fann JI, Hicks GL, et al., eds. Cardiac surgery simulation curriculum: simulation-based training in surgical skills and decision making. Chapel Hill (NC): Thoracic Surgery Directors Association; 2015.

16. Barsness KA, Rooney DM, Davis LM. Collaboration in simulation: the development and initial validation of a novel thoracoscopic neonatal simulator. J Pediatr Surg. 2013;48:1232-8.

Key Words: 3D printing, operative simulation, surgical education, hypertrophic cardiomyopathy, septal myectomy

Readers who found these articles interesting may also like to read the following papers found in recent and future issues of our sister publications, Seminars in Thoracic and Cardiovascular Surgery and Operative Techniques in Thoracic and Cardiovascular Surgery!

Evolving Technology and Basic Science

Original Submission: Creation and Global Deployment of a Mobile, Application-Based Cognitive Simulator for Cardiac Surgical Procedures. Zachary E. Brewer. Semin Thoracic Surg 2016; 28:1-9.

Editorial Commentary: “And” is Better Than “Or.” Joshua L. Hermsen. Semin Thoracic Surg 2016; 28:10-11. 